\title{
Modeling and Control of Needles With Torsional Friction
}

\author{
Kyle B. Reed*, Associate Member, IEEE, Allison M. Okamura, Senior Member, IEEE, \\ and Noah J. Cowan, Member, IEEE
}

\begin{abstract}
A flexible needle can be accurately steered by robotically controlling the bevel tip orientation as the needle is inserted into tissue. Friction between the long, flexible needle shaft and the tissue can cause a significant discrepancy between the orientation of the needle tip and the orientation of the base where the needle angle is controlled. Our experiments show that several common phantom tissues used in needle steering experiments impart substantial friction forces to the needle shaft, resulting in a lag of more than $45^{\circ}$ for a $10 \mathrm{~cm}$ insertion depth in some phantoms; clinical studies report torques large enough to cause similar errors during needle insertions. Such angle discrepancies will result in poor performance or failure of path planners and image-guided controllers, since the needles used in percutaneous procedures are too small for state-of-the-art imaging to accurately measure the tip angle. To compensate for the angle discrepancy, we develop an estimator using a mechanics-based model of the rotational dynamics of a needle being inserted into tissue. Compared to controllers that assume a rigid needle in a frictionless environment, our estimatorbased controller improves the tip angle convergence time by nearly $50 \%$ and reduces the path deviation of the needle by $70 \%$.
\end{abstract}

Index Terms-Biomechanics, feedforward systems, robots, surgery.

\section{INTRODUCTION}

A CCURATE needle tip placement is essential for many needle-based diagnostic and therapeutic procedures, such as biopsies and prostate brachytherapy [1], [2]. Guiding a maneuverable needle in real time can improve placement accuracy, enable access to multiple targets for a single insertion, and expand the range of procedures performed with needle interventions by providing the ability to avoid obstacles and reach previously inaccessible subsurface targets.

One mechanism for steering needles involves harnessing the unbalanced reaction forces imparted by tissue on an asymmetrictip needle as it is inserted into tissue. For sufficiently flexible

Manuscript received March 27, 2009; revised May 29, 2009. First published August 18, 2009; current version published November 20, 2009. This work was supported in part by the National Institutes of Health under Grant R01EB006435. This paper was presented in part at the 2008 IEEE Robotics and Automation Society-Engineering in Medicine and Biology Society International Conference on Biomedical Robotics and Biomechatronics, and the 2009 IEEE International Conference on Robotics and Automation. Asterisk indicates corresponding author.

${ }^{*} \mathrm{~K}$. B. Reed was with the Department of Mechanical Engineering, Laboratory for Computational Sensing and Robotics, The Johns Hopkins University, Baltimore, MD 21218 USA. He is now with the University of South Florida, Tampa, FL 33620 USA (e-mail: reedkb@ alumni.northwestern.edu).

A. M. Okamura and N. J. Cowan are with the Department of Mechanical Engineering, Laboratory for Computational Sensing and Robotics, The Johns Hopkins University, Baltimore, MD 21218 USA (e-mail: aokamura@jhu.edu; ncowan@jhu.edu).

Color versions of one or more of the figures in this paper are available online at http://ieeexplore.ieee.org.

Digital Object Identifier 10.1109/TBME.2009.2029240

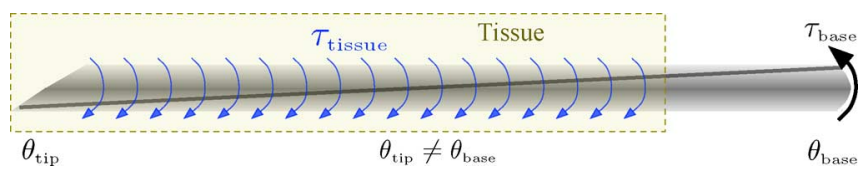

Fig. 1. Torsional friction: Rotating a needle inside tissue can cause the tip angle to deviate from the base angle due to friction along the tissue-needle interface.

needles, these forces cause the needle to follow a nearly circular arc of constant curvature [3]. The instantaneous direction of curvature can be controlled by rotating the needle about its shaft at the base of the needle (outside the tissue), thereby reorienting the needle tip. The needle tip can then be guided to a desired location inside the body while avoiding obstacles. See [4] for a survey that includes robotically steered needles and insertion modeling.

Specifically for prostate brachytherapy, the steerable needle could be precisely driven to a target where a radioactive seed is delivered. Without removing the needle tip from the tissue, the tip can then be steered to subsequent targets for further seed placement. Biopsy procedures typically employ large needles that cannot maneuver inside tissue. In this case, a small steerable needle could accurately reach the target and the larger biopsy needle could reach the target by sliding over the steerable needle. The steerable needle would then be removed and the tissue excised using standard methods.

When rotating (steering) the needle inside tissue, friction between the tissue and the inserted length of the needle results in the angle of the needle tip "lagging" the angle of the needle base, as illustrated in Fig. 1. We show that several tissues impart sufficient frictional forces to the needle shaft to cause significant discrepancies between the base and tip angles. During clinical needle insertions for prostate brachytherapy, Podder et al. [5] found torques large enough to cause a flexible needle to similarly twist in human tissues.

When steering a needle using an asymmetric tip, any inconsistency between base and tip orientations or misalignment of the needle tip can result in poor performance or failure of controllers and path planners. Reed et al. [6] experimentally demonstrate that using a prebent needle tip instead of a bevel-tip needle causes deviations in the tip location when the needle is rotated. The small discrepancy causes the robotically controlled needle to puncture the obstacles and miss the targets. Prebent needles are often used since they afford greater bending due to the larger tip asymmetry.

Current medical imaging technology does not enable direct measurement of the bevel angle around the needle shaft due to the small diameter of the needles used in clinical procedures. 


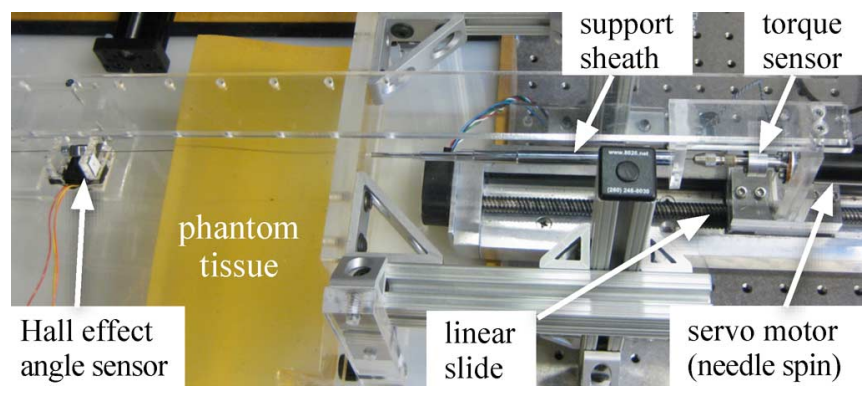

Fig. 2. Experimental setup: The needle is inserted through $10 \mathrm{~cm}$ of plastisol and is attached to a Hall-effect angle sensor. The angle sensor measures the tip angle as the needle moves through the tissue.

Ultrasound and other imaging techniques can achieve a resolution of $0.8 \mathrm{~mm}$ [7], which is similar to the diameter of needles used in many percutaneous procedures. At these resolutions, imaging alone is unable to determine the roll of the bevel tip. Kallem and Cowan [8] detail an image-based state estimation approach to recover the needle shaft orientation in real time, but their controller assumes that changes in the base angle directly relate to changes in the tip angle. They demonstrate that the real system converges slower than simulations, which they speculate is due to unmodeled torsion dynamics. Our torsion controller is designed to work with controllers such as their planar controller to decrease the convergence time.

Several papers [8]-[13] mention the possibility that torsional friction along the inserted portion of the needle could be a problem, but the authors are not aware of any work quantifying or modeling this effect during continuous insertion. Abolhassani et al. [9] and Reed [10] describe a method of adjusting the needle rotation based on the torque measured at the base of the needle while the needle is not moving in translation. Several studies examine the friction required to insert a needle into tissue [14], [15], but the authors are not aware of any previous studies that analyze or model the torsional friction during continuous insertions.

This paper develops a fundamental model of a long thin object rotating inside a continuous medium. We demonstrate the practical significance of torque generated by the needle-tissue interface and formulate a model of the rotational dynamics that can be incorporated into path planners and position controllers for steerable needles like the system described in [6].

\section{QUANTIFYING NEEDLE LAG}

\section{A. Materials and Methods}

We use the device shown in Fig. 2 for our measurements and experimental validation. The setup is capable of rotating and inserting a needle into tissue using two dc servo motors. A sixaxis sensor (ATI Nano 17) measures the forces and torques at the base of the needle.

For the experiments described throughout this paper, we use a solid 0.59-mm-diameter Nitinol wire (Nitinol Devices and Components, Fremont, CA) with a bevel tip, unless stated otherwise. The needle's polar moment of inertia is defined as $J=\pi d^{4} / 32$; therefore, $J=0.012 \mathrm{~mm}^{4}$. We determine the shear modulus $G$ by rigidly fixing the needle tip at a fixed angle while rotating the needle base. The remainder of the needle is unrestrained. The angular change (in radians) over the length $l$, with torque $\tau$, is given by

$$
\theta=\int_{0}^{l} \frac{\tau(x) d x}{J(x) G(x)} .
$$

A $90^{\circ}$ rotation of the $41 \mathrm{~cm}$ needle resulted in a torque of $1.24 \mathrm{~N} \cdot \mathrm{mm}$; therefore, $G$ is $27.2 \mathrm{GPa}$. The density of the Nitinol needle is $6.45 \mathrm{~g} / \mathrm{cm}^{3}$.

To quantify the effect of torsion, we inserted the needle through the five tissue types described in Table I. All tissues, except for the chicken's, were homogenous. We inserted the needle in a straight path by rotating the needle $180^{\circ}$ every $1 \mathrm{~cm}$. A needle inserted in a highly curved path under large torsion would be more likely to buckle [16], but we have not seen this effect during our testing. After inserting, we instrumented the protruding needle tip with two magnets. A Hall-effect sensor (Honeywell Sensing and Control, Golden Valley, MN) measured the angle of the needle tip with a resolution of at least $0.6^{\circ}$ over the range used during these experiments. A platform maintained the proper distance between the Hall-effect sensor and the magnets on the needle during insertion and retraction. The platform and needle translated together. Extending the needle through the tissue eliminates the possible effect of the bevel tip on rotation, thus isolating the effect of shaft friction.

We performed two types of needle rotation experiments: 1) the needle was not translating and 2) the needle was translating. In the nontranslating experiments, the needle was inserted such that the distance between the needle base and the tissue was $23 \mathrm{~cm}$. Each material was tested with a tissue depth of $10 \mathrm{~cm}$, and the porcine gelatin and plastisol were additionally tested at $2.5,5$, and $7.5 \mathrm{~cm}$. One test consisted of rotating the needle base by $90^{\circ}$ and another consisted of rotating the needle base clockwise and counterclockwise by $90^{\circ}$. Both variations ended by retracting the needle $0.5 \mathrm{~cm}$ because we suspected that retracting the needle slightly would break the stiction, allowing the needle to release much of its torsional energy.

For the translating experiment, the needle base started $5 \mathrm{~cm}$ from the tissue, as shown in Fig. 2, and retracted at $0.25 \mathrm{~cm} / \mathrm{s}$. To reduce the effects of the needle buckling inside the support sheath, we retracted the needle instead of inserting it. Buckling would have caused the needle to press against the support sheath and cause a further lag between the tip and the base. The needle tip is not cutting since it is outside the tissue; therefore, the force should be the same during insertion and retraction. Since there is a possibility of added forces from the support sheath during insertion, we chose retraction to focus on the interaction forces. Future needle steering systems could be designed without the problems inherent in this support sheath. The plastisol was tested at depths of 5,10 , and $20 \mathrm{~cm}$.

\section{B. Tip Lag Without Inserting the Needle}

1) Tissue Effects: Torsional friction is a significant problem for all the phantoms listed in Table I. Fig. 3 shows the lag associated with slowly rotating the needle base $90^{\circ}$. The plastisols have a lag of about $10^{\circ}$ and show considerable creep once the 
TABLE I

TISSUE AND PHANTOM DESCRIPTIONS

\begin{tabular}{|l|l|l|}
\hline Material & Description & Vendor \\
\hline \hline Chicken breast & Needle inserted parallel to muscle fibers & Costco, Inc. \\
\hline Soft Plastisol & Fabricated using plastic:softener (stock \# 2228 LP and 2228 S, respectively) of 32:9 & M-F Mfg Co, Inc. \\
\hline Hard Plastisol & Fabricated as above using plastic:softener of 4:1 & M-F Mfg Co, Inc. \\
\hline Porcine gelatin & Fabricated using a ratio of 5 tbsp $\left(74 \mathrm{~cm}^{3}\right)$ of powder (part \# G2500) to $1 \mathrm{cup}\left(237 \mathrm{~cm}^{3}\right)$ of water & Sigma-Aldrich Co. \\
\hline SimTest & Ballistics gel typically used for high velocity impact tests & Corbin, Inc \\
\hline
\end{tabular}

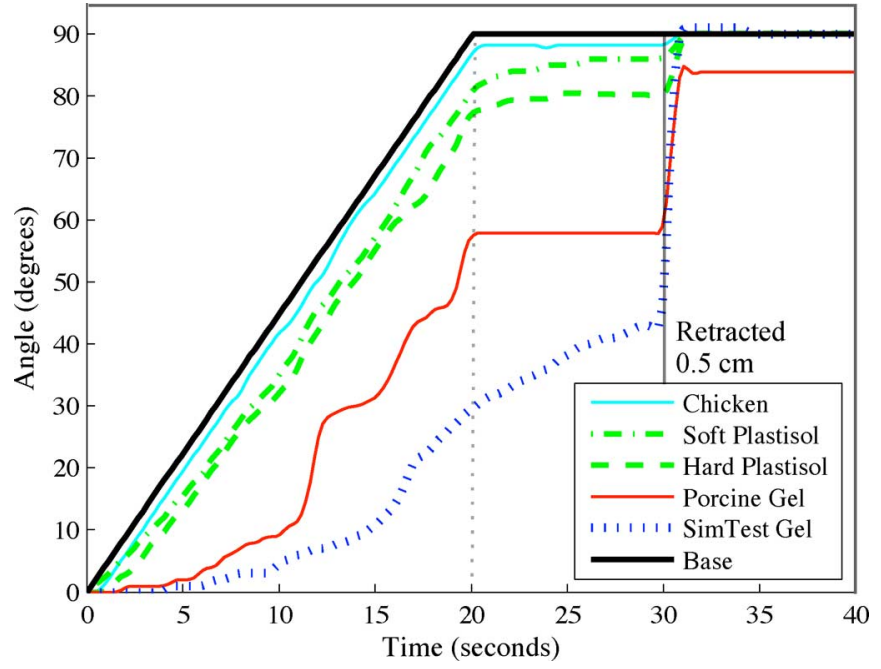

Fig. 3. Torsion behavior in multiple materials: For each material, the needle base was rotated $90^{\circ}$ over $20 \mathrm{~s}$, and then held stationary for $10 \mathrm{~s}$. Each phantom had enough friction to cause significant torsional lag at the tip. The chicken only lagged by $3^{\circ}$. At $t=30 \mathrm{~s}$, the needle was retracted $0.5 \mathrm{~cm}$ to break the stiction, which caused the needle tip to snap to the correct angle in most materials.

base has stopped rotating. SimTest gel shows a similar, yet more significant, creep effect during the same time period. Porcine gelatin exhibits the most dramatic "stick-slip" behavior of the tissue samples tested, which is indicative of substantial stiction; for this material, the needle almost immediately reaches steady state when the base rotation halts. Chicken has tissue structures relatively similar to human tissue, so it may provide results similar to human tissue. However, the chicken tested had been packaged in a fluid that may have significantly reduced its coefficient of friction. A lag of $3^{\circ}$ is not likely to cause significant placement errors, but, as we will discuss in Section II-D, there is likely to be a larger and problematic angle lag in human tissues.

2) Depth Effects: Torsional friction is a function of depth since more tissue interacts with the needle at deeper insertions [15]. Fig. 4 shows the lag associated with rotating the needle base at four different depths in porcine gelatin. As expected, the depth of insertion affects the torsion lag.

The torque measured at the base also increases with the depth during both the constant angular velocity and also once the tip reaches a steady offset. Table II shows the average torque while the needle is not rotating in porcine gelatin $(t=20-30 \mathrm{~s})$ and the damping coefficient during the constant rotation in the plastisol ( $t=10-20 \mathrm{~s})$. The static and damping forces increase linearly with depth. Compared to a least squares fit line, the $R^{2}$ values are 0.98 and 0.97 for porcine gelatin and plastisol, respectively.

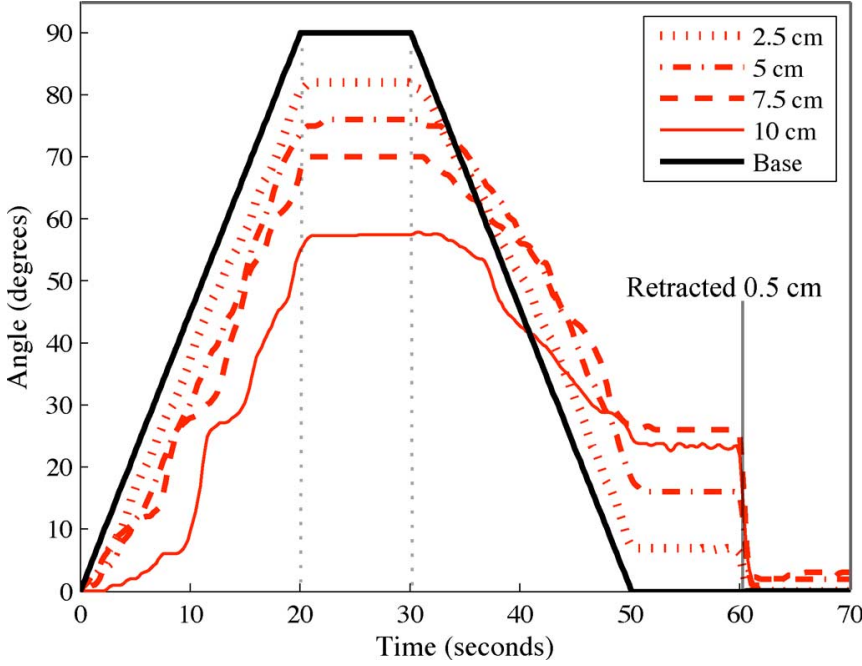

Fig. 4. Torsion behavior for multiple insertion depths: For a single material (porcine gelatin), the needle base was successively rotated $90^{\circ}$ clockwise over 20 $\mathrm{s}$, held stationary for $10 \mathrm{~s}$, and rotated $90^{\circ}$ counterclockwise over $20 \mathrm{~s}$. At $t=$ $60 \mathrm{~s}$, the needle was retracted $0.5 \mathrm{~cm}$. The tip lag increases with increased insertion depth.

TABLE II

ROTATIONAL FRICTION FORCES

\begin{tabular}{|c|c|c|}
\hline $\begin{array}{c}\text { depth } \\
(\mathrm{cm})\end{array}$ & $\begin{array}{c}\text { Mean static force in porcine gelatin } \\
(\mathrm{N}-\mathrm{mm})\end{array}$ & $\begin{array}{c}\text { Viscous damping in plastisol } \\
(\mathrm{N}-\mathrm{mm}-\mathrm{s}) b=\frac{\tau}{\dot{\theta}}\end{array}$ \\
\hline 2.5 & 0.17 & 0.37 \\
\hline 5.0 & 0.32 & 0.94 \\
\hline 7.5 & 0.49 & 1.39 \\
\hline 10.0 & 0.58 & 1.63 \\
\hline
\end{tabular}

Fig. 4 shows other complications associated with torsion windup. At $t=30 \mathrm{~s}$, the base begins to rotate back to $0^{\circ}$ while the tip also starts to move in the same direction as the base. The tip is briefly leading the base. At a depth of $10 \mathrm{~cm}$, the needle tip has rotated by almost $20^{\circ}$ before the base overtakes the tip. This lead effect is likely due to tissue elasticity: the tissue briefly pulls the needle in the same direction as the base. The tissue, rather than the base actuator, is causing the tip to rotate during this early reversal period.

3) Overcoming Torsional Stiction: The final motion in Figs. 3 and 4 is the needle base retracting $0.5 \mathrm{~cm}$. This motion breaks the stiction between the needle and the tissue and allows the needle to snap near to the final base angle. The required retraction distance is a function of the elasticity of the tissue-more elastic materials will require a larger retraction.

One method to overcome the lag is, thus, to retract the needle a small distance after rotating, and then, reinsert the needle at the same distance. Retracting the needle leaves a precut path. 


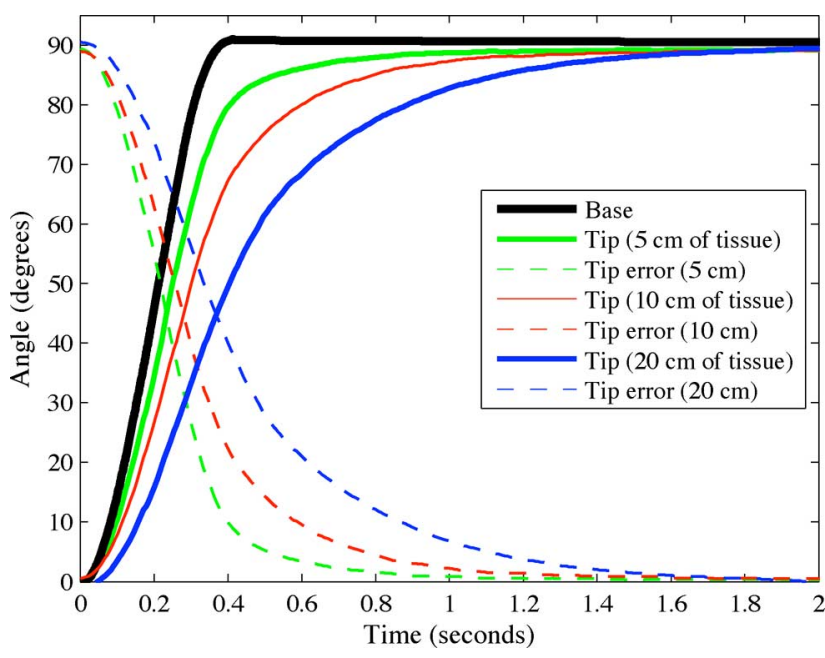

Fig. 5. Torsion behavior during continuous needle motion: For a single material (plastisol), the needle base was quickly rotated $90^{\circ}$ while the needle was retracted at $0.25 \mathrm{~cm} / \mathrm{s}$ through three depths. The tip takes longer to settle to the base angle when inserted through more tissue. The settling time of nearly $2 \mathrm{~s}$ causes significant errors in the needle tip trajectory. Each depth is the average of ten trials.

Upon reinsertion, the needle follows this path and returns to the previous location with the bevel tip at the desired orientation. Additional insertion causes the needle to follow a new path defined by the new tip angle. However, this method is not recommended when using a prebent needle in real tissue since the needle may cause additional tissue damage and follow a different path upon reinsertion.

Another method to reduce the effect of stiction is to use dithering, which adds a high-frequency periodic signal to the insertion [17]. Dithering would keep the controlled surface moving, and thus, prevent the controlled surface from encountering stiction. High-frequency motions are likely unsuitable for needle steering due to the possible additional trauma associated with these vibrations. Some clinicians use a similar strategy as they insert needles by slowly twisting the needle back and forth during insertion.

\section{Tip Lag With Needle Motion}

Even when the needle is continuously moving through the tissue, the tip still lags the base when rotated. Fig. 5 shows the lag associated with rotating a needle in soft plastisol while the needle is being moved through tissue at $0.25 \mathrm{~cm} / \mathrm{s}$. The base motion is tuned to provide the fastest response without oscillating. Similar to the nontranslating case, more tissue contact causes a larger tip lag. Although the nontranslating case remains at a constant offset angle due to static friction, continuously inserting the needle prevents any static friction effects since the needle is constantly sliding through the tissue, so the tip eventually reaches the desired angle.

The insertion velocity used in this experiment is slower than that in a prostate brachytherapy performed by surgeons. However, Podder et al. [5] note that the slower speeds typically used in robotic needle insertions do not significantly affect the procedure compared to the higher velocities used by surgeons. The needle tip will eventually reach the base angle as long as the needle is being inserted fast enough to prevent stiction effects.

Rotating the needle $180^{\circ}$ while inserting causes the needle tip to deviate from its current plane of motion. As the needle rotates about its axis, the tip angle points in an undesired direction; thus, the needle moves away from the desired plane of motion. During the $2 \mathrm{~s}$ it takes for the needle tip to converge to the base angle, the needle will have moved forward and followed a path perpendicular to the desired plane. We demonstrate that this slow convergence causes a needle to deviate by $1.2 \mathrm{~cm}$ over a $7 \mathrm{~cm}$ insertion and develop a model and controller to improve the performance of the tip convergence.

\section{Clinical Implications}

Accurate needle placement is vital to many medical diagnoses and treatments. Even small deviations in the placement of a needle tip during a biopsy can result in misdiagnosis or ineffective placement of radiation during a prostate brachytherapy. Many percutaneous procedures are performed by hand and the forces/torques are not recorded. However, Podder et al. [5] measured the forces and torques in vivo during several prostate brachytherapy needle interventions. Compared to the torque in the other two directions, the torque around the insertion axis appears minuscule, but the $7 \mathrm{~N} \cdot \mathrm{mm}$ they measured is actually higher than the torque we measured in SimTest gel $(1.0 \mathrm{~N} \cdot \mathrm{mm})$, which had a lag of nearly $50^{\circ}$.

Using the torque measured at the base of a needle, Reed [10] demonstrates that the lag between the base and the tip of the needle is calculated as

$$
\theta_{\text {base }}-\theta_{\text {tip }}=\frac{\tau_{\text {base }}}{J G}\left(\frac{l_{\text {in }}+l_{\text {out }}}{2}\right)
$$

where $l_{\text {in }}$ is the distance from the needle base to the entry point in the tissue, $l_{\text {out }}$ is the distance from the needle base to the tip of the needle or the point where the needle exits the tissue, $\tau_{\text {base }}$ is the torque measured at the base of the needle, $G$ is the shear modulus of the needle, and $J$ is the polar moment of inertia of the needle.

Based on (2), the $7 \mathrm{~N} \cdot \mathrm{mm}$ of torque reported by Podder et al. for a human intervention would cause a lag of about $11^{\circ}$ in a $1.27-\mathrm{mm}$-diameter Nitinol needle of the same $20 \mathrm{~cm}$ length inserted $5 \mathrm{~cm}$ into tissue. Podder et al. used larger $1.27-\mathrm{mm}$-diameter stainless steel needles, which reduces the amount of lag due to increased diameter, and thus, torsional stiffness. However, bevel and bent-tip steerable needles require the superelastic properties of materials such as Nitinol. Currently, there is no steering during a prostate brachytherapy since the large stainless steel needles are much more rigid than a steerable needle. When a flexible needle is used to allow the needle to steer, the torques will be large enough to cause significant errors, and it will be necessary to compensate for the developed inaccuracies. Larger steerability will cause larger angle errors, thus increasing the need for torsion compensation. 


\section{DYNAMIC NEEDLE TORSION MODEL}

Currently, it is not possible to measure the angle of the needle tip inside tissue. The only angle measurement is at the base of the needle. At certain instances, the torque at the needle base directly relates to the steady-state lag at the needle tip, as shown in (2), but this equation says nothing about the trajectory of the needle tip. Therefore, in order to estimate the motion of the needle tip for feedback control, we formulate a mechanicsbased model of the rotational dynamics of a needle rotating inside tissue.

The experiments in Section II-C demonstrate that the needle tip eventually reaches the base angle when rotated while translating. Stiction has a minimal effect because the surface of the needle is continuously sliding past the tissue, but the slow angle convergence will cause the needle to move in an unspecified direction. Our model assumes that each part of the needle is moving relative to the local tissue such that the interaction with the tissue is viscous only and the effects of stiction will not be present.

\section{A. Lumped-Mass Model}

As a brute force approach to modeling the needle inserting and rotating inside a tissue, we use a lumped-mass model with viscous friction. This model requires many unnecessary states, but allows us to show that a more compact modal coordinate representation can be used.

Each lumped mass of the needle is connected to neighboring sections by a torsional spring and is connected to the ground through a damper. The dynamics of the lumped-mass system are given by a vector second-order differential equation

$$
\left(\eta l_{s} \boldsymbol{I}_{m \times m}\right) \ddot{\boldsymbol{\theta}}+\left(d \boldsymbol{I}_{m \times m}\right) \dot{\boldsymbol{\theta}}+\boldsymbol{K} \boldsymbol{\theta}=[1,0,0, \ldots, 0]^{T} u(t)
$$

where $\boldsymbol{\theta}$ is a vector of the angles of the $m$ masses, $l_{s}$ is the length between masses, $d$ is the damping exerted on each mass from the needle-tissue interaction, and $u(t)$ is an input exerted only at the first mass. The following terms frequently appear together, so we define $\eta=\rho J$ and $\kappa=J G$, where $\rho$ is the density of the needle and $J$ and $G$ are previously defined needle parameters. The stiffness matrix $\boldsymbol{K}$ is given by

$$
\boldsymbol{K}=\left[\begin{array}{ccccc}
\kappa & -\kappa & & & \mathbf{0} \\
-\kappa & 2 \kappa & -\kappa & & \\
& & \ddots & & \\
& & -\kappa & 2 \kappa & -\kappa \\
\mathbf{0} & & & -\kappa & \kappa
\end{array}\right] \text {. }
$$

For many types of damping, the modal coordinates are coupled through the damping terms and cannot be decoupled into independent states. However, for a proportionally (viscously) damped system, the matrix of eigenvectors decouples the system into modal coordinates [18]. Caughey and O'Kelly [19] show that systems have the same mode shapes (i.e., eigenvectors) as the system without damping if they satisfy

$$
\boldsymbol{K} \boldsymbol{\Lambda}^{-1} \boldsymbol{D}=\boldsymbol{D} \boldsymbol{\Lambda}^{-1} \boldsymbol{K}
$$

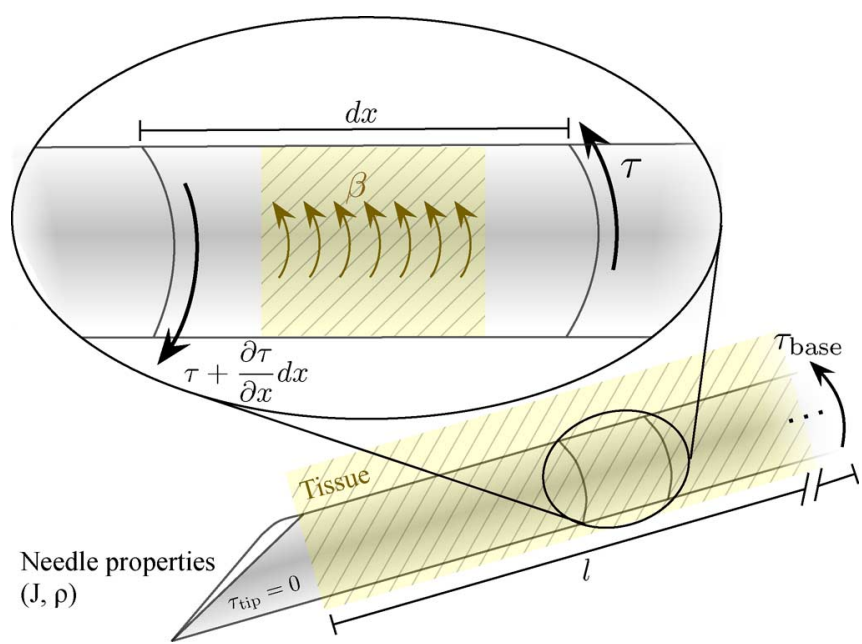

Fig. 6. Needle model: The torque from neighboring elements and the damping from the tissue act on each small element of the needle.

where $\boldsymbol{K}$ is the stiffness matrix, $\boldsymbol{\Lambda}$ is the inertia matrix, and $\boldsymbol{D}$ is the damping matrix.

The system described by 3 is viscously damped and satisfies (5); thus, it can be described conveniently in modal coordinates as linear independent combinations of the normal modes, regardless of the amount of friction. Describing a system in modal coordinates reduces the number of states necessary to adequately describe the system. In Section III-E, we highlight the benefits of the modal model as we compare it with the lumped-parameter model.

\section{B. Continuous Model}

Fig. 6 shows our model of the needle inside tissue and the torque exerted on a small element of the needle. Writing Newton's second law for this small element results in

$$
\frac{\partial \tau}{\partial x} d x-\beta \frac{\partial \theta}{\partial t} d x=\eta \frac{\partial^{2} \theta}{\partial t^{2}} d x
$$

where $\tau$ is the torque exerted on this element from neighbor elements (internal torque), $\beta$ is the viscous damping exerted on a small element, $t$ is the time, and $\theta$ is the angle.

As shown in Section II-B2, the total lag increases with insertion depth since additional tissue interacts with the needle. Assuming homogeneous tissue, the total torsional friction increases linearly since each additional piece of tissue exerts an additional resistive force. Thus, we model the friction at each small element as

$$
\beta=\frac{b}{l}
$$

where $b$ is the effective damping coefficient along the entire needle-tissue interface of length $l$.

Mechanics principles show that the torque required to twist a shaft of length $l$ by an angle $\theta$ is determined by (1). The needle (shaft) has constant material properties; therefore, $J(x)=J$ and $G(x)=G$. Taking the second partial derivative of (1) with 
respect to $x$ results in

$$
\frac{\partial \tau}{\partial x}=\kappa \frac{\partial^{2} \theta}{\partial x^{2}} .
$$

Substituting (8) into (6) and integrating results in a homogenous partial differential equation that defines the motion of the needle through time and space, we get

$$
\kappa \frac{\partial^{2} \theta}{\partial x^{2}}=\eta \frac{\partial^{2} \theta}{\partial t^{2}}+\beta \frac{\partial \theta}{\partial t} .
$$

\section{Forced Modal Analysis}

Modal analysis is often used to analyze many structures such as cantilever beams [18], [20], [21]. The authors, however, are not aware of an analysis on long slender rods being controlled on one edge with distributed damping, such as needles inserted inside tissue. In fact, modal analysis of members in torsion is not studied nearly as extensively as cantilevered beams.

To control the orientation of the needle, a torque is exerted at the base. We represent the torque as a spatial impulse function

$$
v(x, t)=\delta(x) u(t)
$$

where $\delta$ is the Dirac impulse function and $u(t)$ is the input torque. The forced partial differential equation is

$$
\eta \frac{\partial^{2} \theta}{\partial t^{2}}+\beta \frac{\partial \theta}{\partial t}-\kappa \frac{\partial^{2} \theta}{\partial x^{2}}=v(x, t) .
$$

We assume that the solution is separable in time and space, so we can write the solution as a standard Fourier series expansion

$$
\theta(x, t)=\frac{1}{2} \psi_{0}(x) q_{0}(t)+\sum_{k=1}^{\infty} \psi_{k}(x) q_{k}(t)
$$

where $\psi_{k}(x)$ is typically taken as the $k$ th mode shape and $q_{k}(t)$ is the associated time-dependent factor for each mode. ${ }^{1}$ The zeroth mode corresponds to a constant angle along the spatial coordinate, which is directly measured at the base of the needle. The general solution for each mode shape is

$$
\psi_{k}(x)=\cos \left(\omega_{k} x\right)
$$

where $\omega_{k}$ is the frequency of the $k$ th mode and

$$
\omega_{k}=\frac{k \pi}{l}, \quad k=0,1,2,3, \ldots
$$

Plugging (12) into (11) results in

$$
\begin{aligned}
& \frac{1}{2}\left(\eta \psi_{0} \ddot{q}_{0}+\beta \psi_{0} \dot{q}_{0}\right) \\
& \quad+\sum_{k=1}^{\infty}\left\{\eta \psi_{k} \ddot{q}_{k}+\beta \psi_{k} \dot{q}_{k}-\kappa \psi_{k}^{\prime \prime} q_{k}\right\}=v(x, t) .
\end{aligned}
$$

We apply the orthogonality principle by multiplying (15) by an arbitrary mode shape $\left(\psi_{s}\right)$ and integrating over the length.

\footnotetext{
${ }^{1}$ Of course, one does not require that these be mode shapes-any convenient set of orthogonal basis functions will work; we drop the sine terms for reasons that will be clear shortly.
}

Since each mode is orthogonal to every other mode, we have

$$
\int_{0}^{l} \psi_{k}(x) \psi_{s}(x) d x= \begin{cases}\frac{l}{2}, & k=s>0 \\ l, & k=s=0 \\ 0, & \text { otherwise }\end{cases}
$$

and

$$
\int_{0}^{l} \psi_{k}^{\prime \prime}(x) \psi_{s}(x) d x=\left\{\begin{array}{cl}
-\frac{l}{2} \omega_{k}^{2}, & k=s \\
0, & \text { otherwise. }
\end{array}\right.
$$

We use the sifting property of the Dirac function at $x=0$

$$
\int_{0}^{l} v(x, t) \psi_{s}(x) d x=u(t) \int_{0}^{l} \delta(x) \psi_{s}(x) d x=u(t)
$$

which means that any input at the base excites all cosine modes; the sinusoidal modes are not excited with this base input and similarly do not have any effect on the tip angle. The final differential equation for the $k$ th mode is then

$$
\eta \ddot{q}_{k}(t)+\beta \dot{q}_{k}+\kappa \omega_{k}^{2} q_{k}(t)=\frac{2}{l} u(t) .
$$

Truncating to $n$ modes, we have

$$
\boldsymbol{M} \ddot{\boldsymbol{q}}+\boldsymbol{D} \dot{\boldsymbol{q}}+\boldsymbol{K} \boldsymbol{q}=\boldsymbol{P} u(t)
$$

where

$$
\begin{aligned}
\boldsymbol{M} & =\operatorname{diag}(\eta, \eta, \ldots, \eta) \\
\boldsymbol{D} & =\operatorname{diag}(\beta, \beta, \ldots, \beta) \\
\boldsymbol{K} & =\operatorname{diag}\left(0, \kappa \omega_{1}^{2}, \kappa \omega_{2}^{2}, \ldots, \kappa \omega_{n-1}^{2}\right) \\
\boldsymbol{P} & =\frac{2}{l}[1,1, \ldots, 1]^{T} \\
\boldsymbol{q} & =\left[q_{0}(t), q_{1}(t), q_{2}(t), \ldots, q_{n-1}(t)\right]^{T} .
\end{aligned}
$$

Each state $(q)$ in the modal system represents a mode shape, so observability and controllability correspond to the ability to observe and control the modes of the system. The system can be shown to be fully controllable and observable for any number of modes.

\section{Inertialess Modal Model in State-Space Form}

In the case of needle steering, we observe that the system is overdamped and the inertia of the needle may be insignificant compared to the stiffness and damping. To test the effect of needle inertia, we formulate an inertialess system following the same procedure as before, where $\eta$ is considered zero

$$
\begin{aligned}
& \dot{\boldsymbol{x}}=\boldsymbol{A} \boldsymbol{x}+\boldsymbol{B} u \\
& y=\boldsymbol{C q}
\end{aligned}
$$

where

$$
\begin{aligned}
& \boldsymbol{A}=-\boldsymbol{D}^{-1} \boldsymbol{K} \\
& \boldsymbol{B}=\boldsymbol{D}^{-1} \boldsymbol{P} \\
& \boldsymbol{C}=\left[\psi_{0}(l), \psi_{1}(l), \psi_{2}(l), \ldots, \psi_{k-1}(l)\right]
\end{aligned}
$$

where $\boldsymbol{D}, \boldsymbol{K}$, and $\boldsymbol{P}$ are defined in (21). 
TABLE III

COMPARISON OF MODELS FOR 20 CM INSERTION

\begin{tabular}{|c|c|c|c|c|}
\hline \multicolumn{4}{|c|}{ Dominant Eigenvalues $(\mathrm{rad} / \mathrm{sec})$} & \multirow{2}{*}{$\begin{array}{l}\text { Hankel singular } \\
\text { value ratio } \\
(\min / \max )\end{array}$} \\
\hline $\begin{array}{l}\text { Lumped- } \\
(n=64)\end{array}$ & $\begin{array}{c}\text { System } \\
(n=8)\end{array}$ & $\begin{array}{l}\text { Full Modal } \\
\text { System }\end{array}$ & $\begin{array}{c}\text { Inertialess } \\
\text { System }\end{array}$ & \\
\hline 0.000 & 0.000 & 0.000 & 0.000 & 1.000 \\
\hline-2.686 & -2.652 & -2.687 & -2.687 & $5.396 \times 10^{-1}$ \\
\hline-10.74 & -10.20 & -10.75 & -10.75 & $7.377 \times 10^{-2}$ \\
\hline-24.14 & -21.51 & -24.18 & -24.18 & $1.583 \times 10^{-2}$ \\
\hline-42.85 & -34.84 & -42.98 & -42.98 & $1.710 \times 10^{-3}$ \\
\hline
\end{tabular}

\section{E. Model Comparison}

Table III shows the eigenvalues of the lumped-mass model with both 64 and 8 masses and of the fourth-order modal models with and without inertia for a $20 \mathrm{~cm}$ insertion. The slowest poles of the modal model with mass are within $0.001 \%$ of the poles of the inertialess modal model. The eigenvalues of the 64-mass lumped system are within $0.5 \%$ of the modal models. For an eight-mass lumped system, the eigenvalues deviate by more than $10 \%$. The system shows similar results at other depths. Using a modal model in this case allows the system to be more accurately described using fewer states than the lumped-parameter model. Without sacrificing accuracy, the inertialess modal model further simplifies the system representation, which is beneficial for realtime control of the needle tip. Thus, we use the inertialess modal formulation.

The Hankel singular values of a system quantify the contribution of each state to the system behavior. The ratio of the minimum and maximum values provide a good measure of where to truncate the system. Table III shows that a fifthorder system has a minimum/maximum ratio on the order of $10^{-3}$, which means that the fifth mode accounts for only $0.1 \%$ of the system dynamic response. The contribution of the fifth mode decreases for shorter needles, so a fourth-order model is sufficient to adequately describe and control the system. Thus, we use a fourth-order inertialess modal model to control the angle of the needle tip, as described in Section V.

The vital parameter for torsion compensation is the angle at the tip of the needle, so our analysis focuses on the settling time for the tip angle. Settling time is a reasonable comparison since we are mainly focused on the rotations necessary to steer the needle. Slow settling time causes the needle to deviate from the desired path.

\section{NEEDLE-TISSUE INTERACTION MODEL}

The true behavior of a needle during insertion is dependent not only on the needle dynamics, but also on the dynamics of the tissue. We use the finite-element (FE) model shown in Fig. 7 to simulate the tissue properties and the needle-tissue interaction so that we can determine if tissue effects are significant. We assume that the elements are small enough that tissue interaction torques along each element occur discretely at the associated nodes. The angle between each node is based on (1). The properties are constant throughout the needle, so the angle between two neighboring nodes, $i$ and $i+1$, is

$$
\theta_{i+1}-\theta_{i}=\frac{\tau l}{J G}
$$

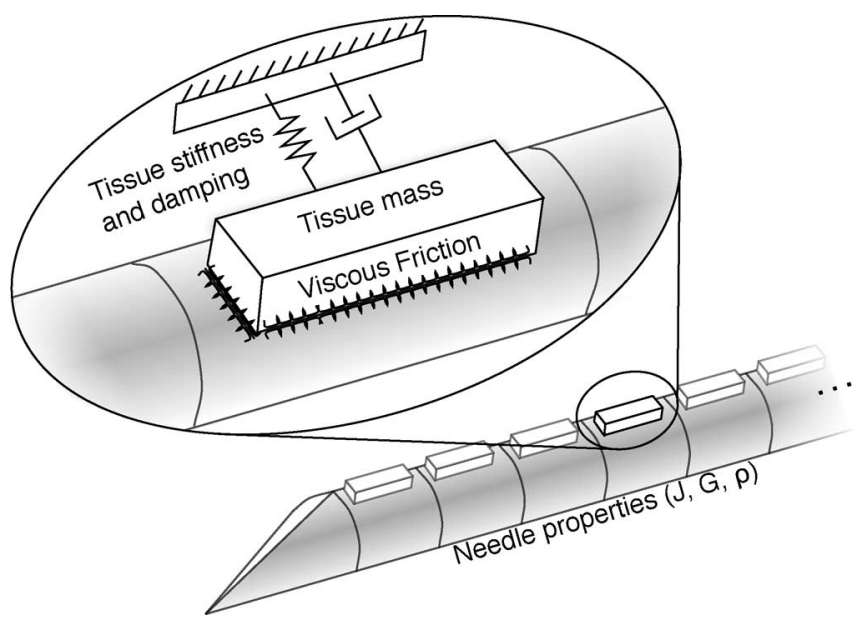

Fig. 7. FE model: The needle-tissue interaction is simulated as viscous friction and the tissue is simulated using the Kelvin-Voigt model.

where $\tau$ is the torque exerted from neighboring nodes and the damping along the element, $l$ is the length between nodes, and $J$ and $G$ are the needle's material properties determined in Section II-A. The input torque is exerted only to the base node and tissue friction is exerted along each element as described in Section IV-A1.

The simulated needle properties and lengths match the experimental setup for the $20 \mathrm{~cm}$ insertion described in Section II-C. The FE model was constructed of 200 disk elements inside the tissue, each of length $1 \mathrm{~mm}$, and one element of length $50 \mathrm{~mm}$ outside the tissue. The element outside the tissue was simply treated as a long torsional member since no external forces acted along its length. The simulation was run with a time step of $10^{-7} \mathrm{~s}$. Decreasing the time step and/or elements does not significantly change the results.

\section{A. Material Characteristics}

1) Needle-Tissue Interaction: In previous studies [10], [15], the interaction between the needle and the tissue was modeled as Karnopp friction, which is composed of dynamic friction everywhere except for a constant static friction within a small velocity near zero. As demonstrated in Fig. 5, the needle tip eventually reaches the desired angle. Static friction causes the needle to maintain a constant offset. Since the needle is constantly inserted through the tissue, the static friction region is never reached, so we model the dynamic friction as viscous damping.

We experimentally determine the viscous damping. During steady-state needle rotation, all applied torque from the tissue is from damping, so we can use

$$
\tau_{b}=b \dot{\theta}
$$

to estimate the damping coefficient $b$. Equation (25) corresponds to the zeroth mode from the modal model (see Section III-C). As shown in Table II, the total rotational damping force increases linearly with depth, much like the way insertion force increases linearly with depth [15]. For the $20 \mathrm{~cm}$ insertion modeled here, we use a total damping coefficient of $b=3.45 \mathrm{~N} \cdot \mathrm{mm} \cdot \mathrm{s}$. The 
total damping is divided equally between each of the elements inside the tissue, so the torque exerted due to friction at each needle element interacting with the tissue, $\tau_{f_{i}}$, is

$$
\tau_{f_{i}}=\frac{b}{n}\left(\dot{\theta}_{i}-\dot{\phi}_{i}\right)
$$

where $\dot{\phi}_{i}$ is the angular velocity of the tissue touching the $i$ th element and $n$ is the number of nodes inside the tissue.

2) Tissue Model: To simulate the tissue properties, we use the Kelvin-Voigt model, which uses a spring and a damper in parallel to model the creep characteristics of tissue [22]. We use the Rheometrics Solids Analyzer II (RSA) experiments performed by Misra et al. [23] for a similar plastisol gel to determine the tissue stiffness and damping parameters. The RSA test measures the force as a displacement is applied to a known surface area of the material. This method determines the elasticity and creep of the tissue. Misra et al. show that the tissue elasticity $\left(E_{t}\right)$ is $45.2 \mathrm{kPa}$ and Poisson's ratio is 0.45 . Mechanics principles show that the shear modulus of the tissue $\left(G_{t}\right)$ is calculated as

$$
G_{t}=\frac{E_{t}}{2(1+\nu)}=15.6 \mathrm{kPa} .
$$

As the needle rotates inside the tissue, some of the soft tissue also moves. For this simulation, we assume that the needle rotation affects tissue up to $0.5 \mathrm{~mm}$ away, which is roughly the size of the needle diameter. We then use the shear modulus to approximate the tissue as a spring surrounding each element of the needle. For a force applied perpendicular to the tissue (such as the needle rotating), mechanics principles show that

$$
\frac{F}{\Delta x}=\frac{G_{t} A}{h}
$$

where $F$ is the applied force from the tissue, $\Delta x$ is the distance the surface of the tissue moves, $h$ is the assumed height of the affected tissue, and $A$ is the surface area of the needle-tissue interface. $F / \Delta x$ is an equivalent spring for the tissue over each element. Using the surface area of each needle element inside tissue $\left(A=1.9 \mathrm{~mm}^{2}\right)$, the interaction height $(h=0.5 \mathrm{~mm})$, and the radius of the needle $(r=0.295 \mathrm{~mm})$, the tissue surrounding each node of the needle is approximated by a rotational spring $\left(k_{t}\right)$ where

$$
k_{t}=\frac{F r^{2}}{\Delta x}=5.2 \times 10^{-3} \frac{\mathrm{N} \cdot \mathrm{mm}}{\mathrm{rad}}
$$

The RSA test linearly increases the strain on the tissue for $5 \mathrm{~s}$ and then maintains a constant position for $5 \mathrm{~s}$. During the $5 \mathrm{~s}$ at a constant position, the measured force decreases as the material creeps with a time constant of 0.96. Since the KelvinVoigt model is a spring and a damper in series, the time constant $a$ is

$$
a=\frac{k_{t}}{b_{t}}
$$

where $b_{t}$ is the tissue damping. The damping coefficient $\left(b_{t}\right)$ for each element is then solved to be $5.4 \times 10^{-3} \mathrm{~N} \cdot \mathrm{mm} \cdot \mathrm{s} / \mathrm{rad}$.

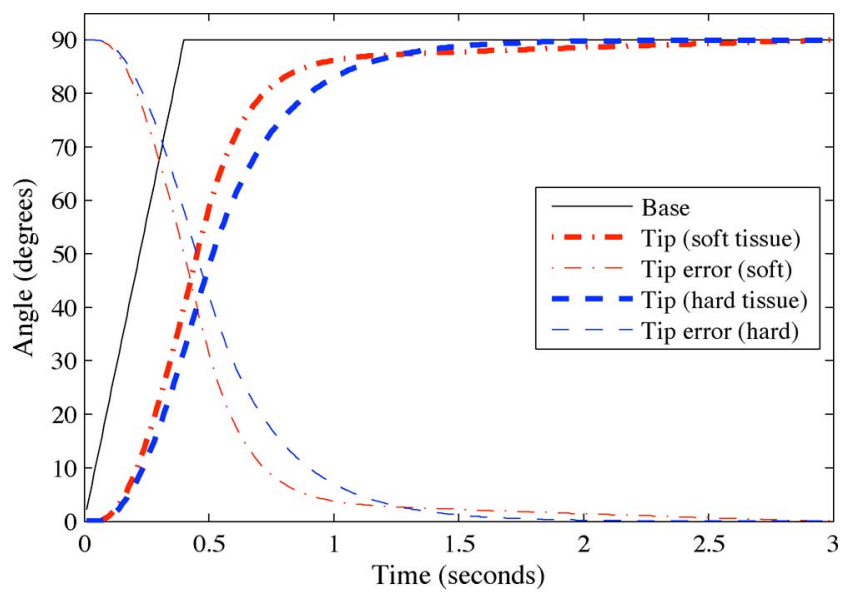

Fig. 8. FE simulation: The hard tissue shows little effects of tissue. The soft tissue initially rotates faster, but does not reach the desired angle as quickly.

\section{B. FE Simulation}

We ran two simulations of the needle with all parameters held constant except for the stiffness and damping of the tissue. We ran the FE model with an input similar to that used in the experimental tests. Fig. 8 shows the tip angles for the two simulated tissue types: one hard and one soft. The soft tissue is modeled after the soft plastisol gel described in Section IV-A, and the hard tissue has a stiffness and damping coefficient 100 times larger than the modeled plastisol, which is much stiffer than any tissue a steerable needle would be inserted through.

The needle tip rotates slightly differently in the soft and hard tissues. The needle in soft tissue initially rotates faster than in the hard tissue because the soft tissue moves slightly with the needle, thus providing less impedance. The simulated soft tissue rotates to within $3^{\circ}$ in $1.05 \mathrm{~s}$ and the hard tissue in $1.22 \mathrm{~s}$, a difference of $15 \%$, but the needle in soft tissue does not reach the desired angle for several seconds.

The soft tissue starts to creep back toward its original location as the needle approaches the desired angle. As the tissue returns, it pulls the needle slightly away from the base angle, causing the needle to briefly maintain a small offset. The tissue reaches its resting state around $2.7 \mathrm{~s}$, and the needle then reaches the desired angle. For multiple rotations in alternating directions, the restoring force of the tissue applies a torque in the direction of the second rotation and briefly aids in needle rotation, much like the multiple rotation experiments described in Section II-B2 and in [10]. Overshooting the desired angle can alleviate much of tissue effect since the final motion causes the tissue to return to its resting state as the needle reaches the desired angle.

Another metric to determine the impact of the angle lag is the integral of the tip error during a rotation. This integral indicates how much the needle diverges from the desired path. The integral of the error in the soft tissue is $43.8 \mathrm{~s} \cdot{ }^{\circ}$ and the hard tissue is $48.6 \mathrm{~s} \cdot{ }^{\circ}$, a difference of $10 \%$.

Although tissue elasticity causes a $10 \%-15 \%$ difference in rotation between a soft and a hard tissue, it is unlikely to cause a significant deviation in human tissue. One of the target regions 


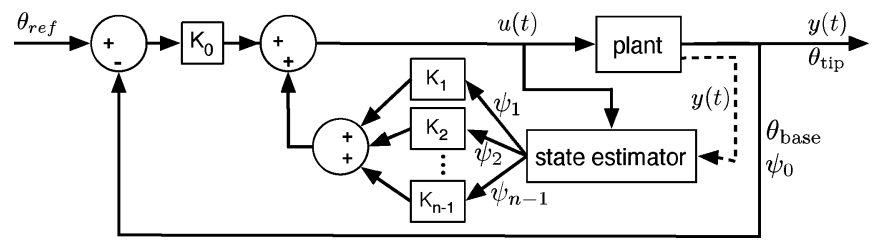

Fig. 9. Torsion controller: The modeled dynamics are estimated and used in state feedback. We also compared the open-loop estimator with a feedback estimator (dotted line).

for needle steering is the prostate, which Krouskop et al. [24] shows has a Young's modulus of around $60 \mathrm{kPa}$, which is larger than that of the tissue used in our simulations. The higher stiffness in in vivo tissue will reduce the effects of tissue elasticity when a needle is rotated. Since the controller we discuss next naturally develops a small overshoot and the tissues are likely to be stiffer than those tested here, we do not foresee tissue elasticity causing a significant angle difference. Any remaining effects from the soft tissue can be eliminated by assuming a slightly more flexible needle to account for tissue elasticity. We will assume no tissue effects and will use the continuous model for the remainder of this paper.

\section{Control of the NeEdle TiP}

The fourth-order inertialess modal model without tissue effects described in Section III-C allows better control of the needle tip than using the base angle measurement alone. Since there is no feedback of the needle tip angle, we will use a state estimator to predict the current states (mode shapes) of the needle.

For comparison, we develop two estimators: 1) feedforward and 2) feedback from the tip angle sensor described in Section II-A. The accompanying controller is designed to control the modes of the system, as opposed to the typical method of controlling positions or velocities of discrete points. The block diagram for the estimator-controller is shown in Fig. 9. The dotted line indicates the tip angle feedback that is not available during a needle insertion. In both observers, the zeroth mode is the needle angle directly measured at the needle base and the higher modes are estimated based on the derived dynamics. The control input is specified based on the estimated position of the needle tip, which is the sum of each mode evaluated at the needle tip. Without feedback, the nonzero-mode system dynamics are estimated as

$$
\begin{aligned}
\dot{\hat{\boldsymbol{x}}} & =\boldsymbol{A} \hat{\boldsymbol{x}}+\boldsymbol{B} u \\
\hat{y} & =\boldsymbol{C} \hat{\boldsymbol{x}}
\end{aligned}
$$

where $\boldsymbol{A}, \boldsymbol{B}, \boldsymbol{C}$, and $\boldsymbol{x}$ are defined in (23).

In the comparative case with tip feedback, we used a Luenberger observer

$$
\dot{\hat{\boldsymbol{x}}}=\boldsymbol{A} \hat{\boldsymbol{x}}+\boldsymbol{B} u+\boldsymbol{L}(y-\hat{y})
$$

where the state matrices are the same as 31 and the feedback gains $(\boldsymbol{L})$ were chosen so that the eigenvalues of $\boldsymbol{A}-\boldsymbol{L} \boldsymbol{C}$ are five times faster than the system $(\boldsymbol{A})$ eigenvalues.
We used the same needle described in Section II-A with the damping values determined from Table II. Fig. 10 shows the needle base and average tip trajectories resulting from three trials of a rotation with and without torsion compensation at two depths. The torsion compensator is able to rotate the needle tip to within $3^{\circ}$ in nearly half the time of only rotating the base to the desired angle. The needle tip convergence times are shown in Table IV. The benefit of compensating for torsion increases at deeper insertions, since the total friction, and thus, the tip lag, increases with the distance inserted. The overshoot in the base angle alleviates much of the tissue effects since the final motion is in the same direction as the tissue relaxation.

The feedback estimator is faster than the feedforward estimator. Although no tip feedback is available for the rotation angle during a needle insertion, comparing the two estimators shows that the feedforward mechanics-based model is capable of correcting a large portion of the tip angle error.

The integral of the tip angle error during the rotations indicates how slow needle tip convergence affects the path. Table $\mathrm{V}$ shows the integral of the tip angle error over $2 \mathrm{~s}$ for the needle with and without torsion compensation. Note that Fig. 10 shows the absolute value of the tip error, but this integral incorporates negative angles because a negative angle will counteract the out-of-plane motion caused by a positive angle. Adding torsion compensation decreases the integral error by about $40 \%$. A control scheme could be to drive this error to zero, ensuring minimal motion out of the plane.

The faster convergence time and decreased tip error with torsion compensation allow more accurate control of the needle position in the tissue. We tested the feedforward torsion compensation by inserting a needle $7 \mathrm{~cm}$ at $0.5 \mathrm{~cm} / \mathrm{s}$. We used a prebent needle with a $45^{\circ}$ bevel and a $15^{\circ}$ prebend $12 \mathrm{~mm}$ from the tip in a complimentary direction. Reed et al. [6] experimentally determined the radius of curvature to be $6.1 \mathrm{~cm}$. Fig. 11 shows the final positions from the camera for each case. The baseline case consists of an insertion without the needle rotating. The baseline was run twice: 1) with the needle curved to the right and 2) with the needle rotated $180^{\circ}$ before insertion so that the needle curved to the left. In each of these baseline trials, the needle did not drastically deviate from the starting plane. In the second case, the needle base rotated $180^{\circ}$ after each of the first $4 \mathrm{~cm}$ without torsion compensation. After a $7 \mathrm{~cm}$ insertion, the needle had deviated by $12 \mathrm{~mm}$ out of the plane. The same four rotations with torsion compensation only caused a $4 \mathrm{~mm}$ deviation over a $7 \mathrm{~cm}$ insertion. Fig. 12 shows the height of the needle for each case. Each point represents the triangulated and filtered deviation from the desired plane recorded at $7.5 \mathrm{~Hz}$. All cases started at the same point, but the cameras were unable to accurately track the tip during the first centimeter due to edge occlusions in the tissue.

Although the torsion compensation does not take into account the added torques caused by the prebent tip, the controller was able to reduce the height deviation by nearly $70 \%$. A bevel-tip needle has less curvature, so the deviations from a plane would be smaller, but would show a similar proportion of deviation with and without torsion compensation. The rotations in this example could have alternated directions between the tip pointing up and 


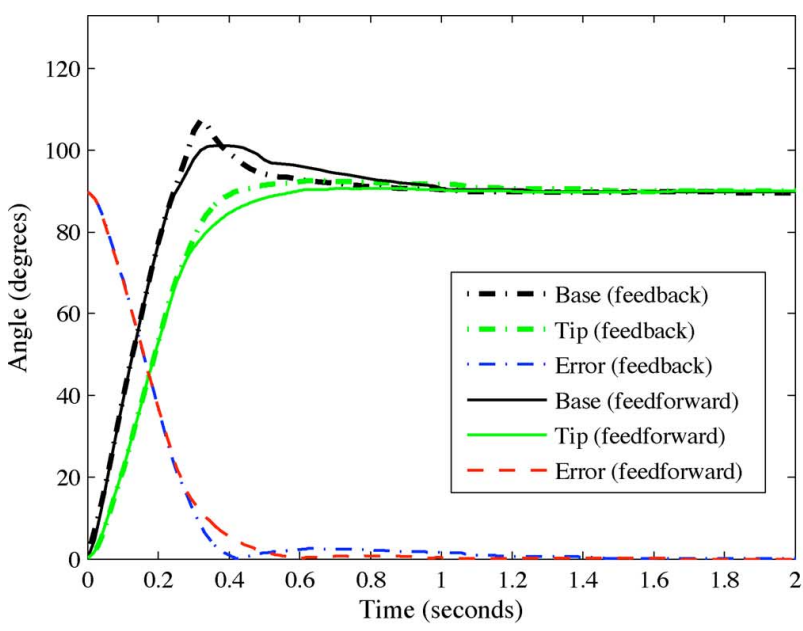

(a)

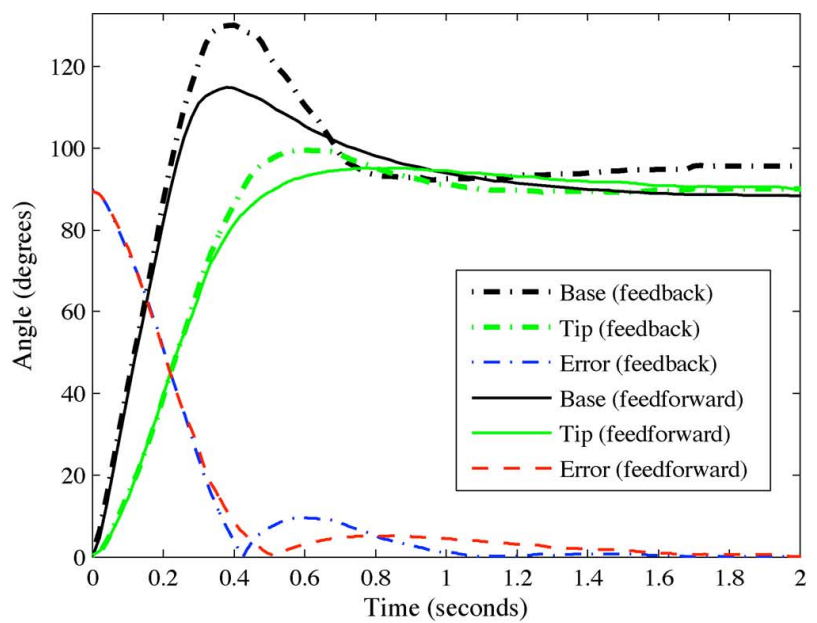

(b)

Fig. 10. Estimator-controller with feedback and feedforward: Experimental results showing that rotating the base of the needle with torsion compensation causes

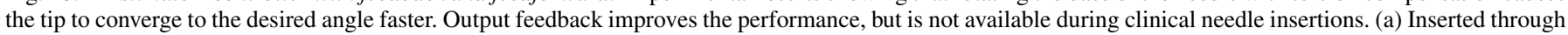
$5 \mathrm{~cm}$ of tissue. (b) Inserted through $10 \mathrm{~cm}$ of tissue.

TABLE IV

NEEDLE TIP SETTLING TIME

\begin{tabular}{|c|c|c|c|c|c|}
\hline distance & base only & \multicolumn{2}{|c|}{ feedforward comp. } & \multicolumn{2}{c|}{ feedback comp. } \\
inserted & control (sec.) & sec. & \% faster & sec. & $\%$ faster \\
\hline $5 \mathrm{~cm}$ & 0.67 & 0.46 & 31.8 & 0.36 & 45.8 \\
\hline $10 \mathrm{~cm}$ & 1.02 & 0.51 & 50.0 & 0.41 & 60.1 \\
\hline
\end{tabular}

TABLE V

INTEGRAL OF THE TIP ANGLE ERROR

\begin{tabular}{|c|c|c|c|c|c|}
\hline $\begin{array}{c}\text { distance } \\
\text { inserted }\end{array}$ & base only & \multicolumn{2}{|c|}{ feedforward comp. } & \multicolumn{2}{c|}{ feedback comp. } \\
control $\left(\mathrm{sec}^{\circ}\right)$ & $\mathrm{sec}^{\circ}$ & $\%$ decrease & $\mathrm{sec}^{\circ}$ & $\%$ decrease \\
\hline $5 \mathrm{~cm}$ & 27.0 & 17.2 & 36.4 & 15.3 & 43.6 \\
\hline $10 \mathrm{~cm}$ & 37.2 & 19.0 & 48.9 & 16.6 & 55.3 \\
\hline
\end{tabular}
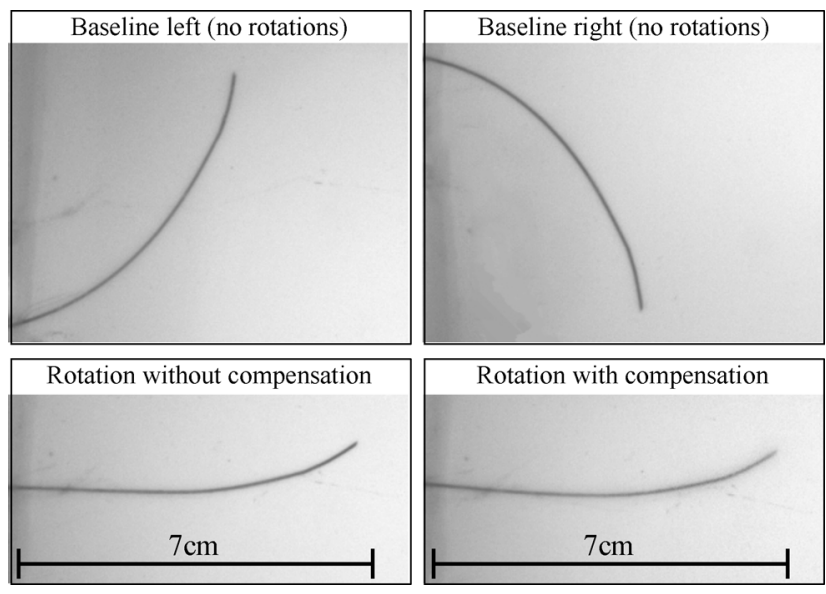

Fig. 11. Insertions with and without torsion compensation: The two experimental insertions shown on top have no needle rotations, so the needle curved in one direction. The insertion shown on the bottom left had four $180^{\circ}$ rotations without torsion compensation. Notice that the tip gets darker toward the right side, indicating the needle coming up out of the page. The insertion shown on the bottom right had four rotations with torsion compensation and remained near the plane of the baseline cases.

the tip pointing down. Alternating the rotation direction would reduce the height deviation, but this example demonstrates the reduced deviation from the plane using torsion compensation.

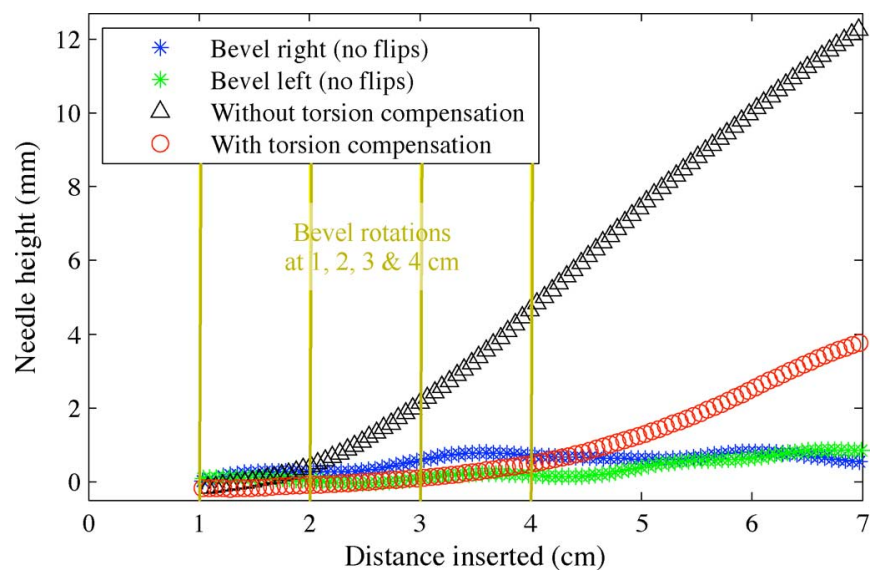

Fig. 12. Needle height deviation: We experimentally inserted the needle without any rotations in two baseline cases. When rotated four times without torsion compensation, the needle drastically deviated from the starting height. With torsion compensation, the needle deviation was greatly reduced.

\section{CONCLUSION}

Friction is detrimental to accurate control of steerable needles, both in the phantom tissues tested here and in human tissues, particularly when using superelastic needles to enhance steering. Torsional friction causes a discrepancy of more than $45^{\circ}$ between the base and tip angles in certain phantom materials. Based on previous experiments with steel needles during a prostate brachytherapy [5], we estimate that torsion will cause a $10^{\circ}-15^{\circ}$ discrepancy in human tissues. It is possible that torsional forces could be amplified by lateral forces on the needle during insertion into human tissue. Such significant errors will likely imperil image-guided controllers and path planners designed for flexible needle steering. Unfortunately, there is a tradeoff between the flexibility of needles for steering and the stiffness of needles to rotate as a rigid body inside tissue. Our model and controller for the torsional dynamics can alleviate 
a large portion of the angle lag due to torsional friction, thus allowing the use of more flexible needles with enhanced steerability.

We developed an estimator-controller based on a modal model of the needle dynamics to predict the tip angle for a needle rotating inside tissue. The controller quickly drives the needle tip to the desired angle. The faster convergence allows the needle to maintain motion in a prescribed plane significantly better than that in the case without torsion compensation. Our torsion model is designed to work in conjunction with the planar controller derived by Kallem and Cowan [8]. They demonstrate that the real system takes twice as long to converge than simulations, which they speculate is due to unmodeled torsion dynamics. Incorporating the torsion dynamics developed here is likely to significantly increase the convergence speed. Future experiments using in vivo tissues with multiple tissue types and membrane layers under medical imaging will be necessary for complete validation.

If a steerable needle or a catheter is controlled by a robot or a clinician via teleoperation, estimating the torque may be important. Clinicians steering a needle will likely occasionally stop and rotate before further insertion. When they subsequently insert further, the inaccuracies following a rotation can cause path deviations. It may be necessary to automatically compensate or provide torque feedback to the doctor. One feedback mode might be an amplified version of the torsional stiffness. This would be particularly important for prebent needles that are not being inserted at that instant.

\section{ACKNOWLEDGMENT}

The authors thank S. Misra, J. Swensen, and T. Wedlick for help with this research.

\section{REFERENCES}

[1] S. Nath, Z. Chen, N. Yue, S. Trumpore, and R. Peschel, "Dosimetric effects of needle divergence in prostate seed implant using I and Pd radioactive seeds," Med. Phys., vol. 27, pp. 1058-1066, May 2000.

[2] J. Youk, E. Kim, M. Kim, J. Lee, and K. Oh, "Missed breast cancers at US-guided core needle biopsy: How to reduce them," RadioGraphics, vol. 27, pp. 79-94, Jan./Feb. 2007.

[3] R. Webster, III, J. S. Kim, N. J. Cowan, G. S. Chirikjian, and A. M. Okamura, "Nonholonomic modeling of needle steering," Int. J. Robot. Res., vol. 25 , no. $5 / 6$, pp. 509-525, 2006.

[4] N. Abolhassani, R. Patel, and M. Moallem, "Needle insertion into soft tissue: A survey," Med. Eng. Phys., vol. 29, pp. 413-431, 2007.

[5] T. Podder, D. Clark, J. Sherman, D. Fuller, E. Messing, D. Rubens, J. Strang, L. Liao, W. Ng, and Y. Yu, "In vivo motion and force measurement of surgical needle intervention during prostate brachytherapy," Med. Phys., vol. 33, no. 8, pp. 2915-2922, 2006.

[6] K. B. Reed, V. Kallem, R. Alterovitz, K. Goldberg, A. M. Okamura, and N. J. Cowan, "Integrated planning and image-guided control for planar needle-steering," in Proc. IEEE Conf. Biomed. Robot., Oct. 2008, pp. 819824.

[7] M. Ding and H. Cardinal, "Automatic needle segmentation in threedimensional ultrasound image using two orthogonal two-dimensional image projections," Med. Phys., vol. 30, no. 2, pp. 222-234, 2003.

[8] V. Kallem and N. J. Cowan, "Image guidance of flexible tip-steerable needles," IEEE Trans. Robot., vol. 25, no. 1, pp. 191-196, Feb. 2009.

[9] N. Abolhassani, R. Patel, and F. Ayazi, "Minimization of needle deflection in robot-assisted percutaneous therapy," Int. J. Med. Robot. Comput. Assist. Surg., vol. 3, pp. 140-148, 2007.
[10] K. B. Reed, "Compensating for torsion windup in steerable needles," in Proc. IEEE Conf. Biomed. Robot., 2008, pp. 936-941.

[11] R. Alterovitz, A. Lim, K. Goldberg, G. S. Chirikjian, and A. M. Okamura, "Steering flexible needles under Markov motion uncertainty," in Proc. IEEE/RSJ Int. Conf. Intell. Robots Syst. (IROS), 2005, pp. 120-125.

[12] R. Webster, III, J. Memisevic, and A. M. Okamura, "Design considerations for robotic needle steering," in Proc. IEEE Int. Conf. Robot. Autom. (ICRA), 2005, pp. 3588-3594.

[13] V. Kallem, D. Chang, and N. J. Cowan, "Task-induced symmetry and reduction in kinematic systems with application to needle steering," in Proc. IEEE/RSJ Int. Conf. Intell. Robots Syst. (IROS), 2007, pp. 3302 3308.

[14] H. Kataoka, T. Washio, K. Chinzei, K. Mizuhara, C. Simone, and A. M. Okamura, "Measurement of the tip and friction force acting on a needle during penetration," in Proc. Med. Image Comput. Comput. Assist. Intervent. (MICCAI) Conf., 2002, pp. 216-223.

[15] A. M. Okamura, C. Simone, and M. O'Leary, "Force modeling for needle insertion into soft tissue," IEEE Trans. Biomed. Eng., vol. 110, no. 51, pp. 1707-1716, Oct. 2004.

[16] J. M. T. Thompson and A. R. Champneys, "From helix to localized writhing in the torsional post-buckling of elastic rods," in Proc. Math., Phys. Eng. Sci., 1996, vol. 452, pp. 117-138.

[17] H. Olsson, K. Åström, C. Canudas De Wit, M. Gäfvert, and P. Lichinsky, "Friction models and friction compensation," Eur. J. Control, vol. 4, no. 3, pp. 176-195, 1998.

[18] D. Inman, Vibration With Control. Chichester, U.K.: Wiley, 2006.

[19] T. Caughey and M. O'Kelly, "Classical normal modes in damped linear dynamic systems," J. Appl. Mech., vol. 32, pp. 583-588, 1965.

[20] A. Salehi-Khojin, S. Bashash, and N. Jalili, "Modeling and experimental vibration analysis of nanomechanical cantilever active probes," $J$. Micromech. Microeng., vol. 18, pp. 085008-1-085008-11, 2008.

[21] S. Bashash, A. Salehi-Khojin, and N. Jalili, "Forced vibration analysis of flexible Euler-Bernoulli beams with geometrical discontinuities," in Proc. Amer. Control Conf., Jun. 2008, pp. 4029-4034.

[22] S. Misra, K. T. Ramesh, and A. M. Okamura, "Modeling of tool-tissue interactions for computer-based surgical simulation: A literature review," Presence: Teleoperators Virtual Environ., vol. 17, pp. 463-491, 2008.

[23] S. Misra, "Realistic tool-tissue interaction models for surgical simulation and planning," Ph.D. thesis, The Johns Hopkins University, 2009.

[24] T. Krouskop, T. Wheeler, F. Kallel, B. Garra, and T. Hall, "Elastic moduli of breast and prostate tisues under compression," Ultrason. Imag., vol. 20, pp. 260-274, 1998.

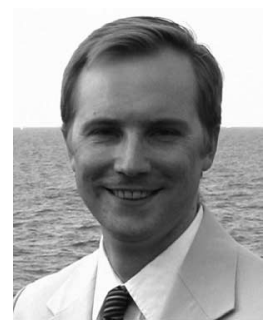

Kyle B. Reed (S'05-A'07) received the B.S. degree from the University of Tennessee, Knoxville, in 2001 , and the M.S. and Ph.D. degrees from Northwestern University, Evanston, IL, in 2004 and 2007, respectively, all in mechanical engineering.

From 2007 to 2009, he was a Postdoctoral Fellow in the Laboratory for Computational Sensing and Robotics, The Johns Hopkins University, Baltimore, MD. In 2009, he joined the faculty of the University of South Florida, Tampa, as an Assistant Professor. His current research interests include haptics, human-machine interaction, rehabilitation engineering, medical robotics, and engineering education.

Prof. Reed was a recipient of the 2001 National Science Foundation Graduate Fellowship. 


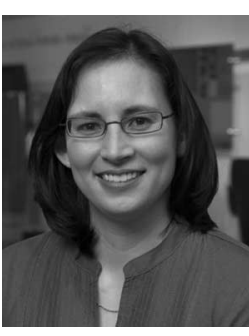

Allison M. Okamura (S'98-A'00-M'03-SM'09) received the B.S. degree from the University of California, Berkeley, in 1994, and the M.S. and $\mathrm{Ph} . \mathrm{D}$. degrees from Stanford University, Stanford, CA, in 1996 and 2000, respectively, all in mechanical engineering.

She is currently a Professor of mechanical engineering at The Johns Hopkins University, Baltimore, $\mathrm{MD}$, where she is also the Decker Faculty Scholar. Her research interests include haptics, teleoperation, robot-assisted surgery, tissue modeling and simulation, rehabilitation robotics, and prosthetics.

Prof. Okamura received the 2004 National Science Foundation CAREER Award, the 2005 IEEE Robotics and Automation Society Early Academic Career Award, and the 2009 IEEE Technical Committee on Haptics Early Career Award. She is an Associate Editor of the IEEE TRANSACTIONS ON HAPTICS.

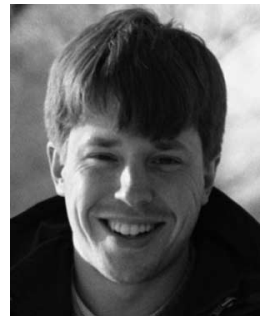

Noah J. Cowan (S'99-M'01) received the B.S. degree in electrical engineering from Ohio State University, Columbus, in 1995, and the M.S. and Ph.D. degrees in electrical engineering and computer science from the University of Michigan, Ann Arbor, in 1997 and 2001, respectively.

From 2001 to 2003, he was a Postdoctoral Fellow at the University of California, Berkeley. In 2003, he joined the faculty of The Johns Hopkins University, Baltimore, MD, as an Assistant Professor. His current research interests include multisensory control in animals and machines.

Prof. Cowan received the National Science Foundation CAREER award in 2009, the William H. Huggins Award for excellence in teaching in 2004, and a Rackham Doctoral Fellowship from the University of Michigan in 2000. 